PROCEEDINGS OF THE

AMERICAN MATHEMATICAL SOCIETY

Volume 129, Number 7, Pages 2157-2162

S 0002-9939(00)05767-1

Article electronically published on November 21, 2000

\title{
FIXED POINTS FOR CONVEX CONTINUOUS MAPPINGS IN TOPOLOGICAL VECTOR SPACES
}

\author{
YU-QING CHEN
}

(Communicated by Alan Dow)

\begin{abstract}
We prove the following result. Let $C$ be a convex compact subset in a topological vector space, and $T: C \rightarrow C$ a convex continuous mapping. (See Definition 1.1.) Then $T$ has a fixed point. Moreover, continuous mappings that can be approximated by convex continuous mappings also have the fixed point property.
\end{abstract}

\section{INTRODUCTION}

The following result is the well-known Schauder fixed point theorem:

Theorem (25]). Let $C$ be a convex compact subset in a normed space, and $T$ : $C \rightarrow C$ be a continuous mapping. Then $T$ has a fixed point in $C$.

This theorem has been generalized to locally convex spaces by many authors for various types of mappings. See, e.g., Tychonoff [27], Browder [1], Fan [5], Glicksberg [6], Himmelberg [10], Reich [19], [20]. The following is an outstanding problem. See 7].

Schauder's conjecture. Let $C$ be a convex compact subset in a topological vector space. Then a continuous mapping $T: C \rightarrow C$ has a fixed point.

Many mathematicians have studied this problem and some progress has been made in topological vector spaces with some special structure. See Klee [14], Zima 29], Rzepecki [24], Hadzic 87, 9], Idzik [11, Nguyen [16, 17], Nguyen and Le [18]. However, this problem still remains unsolved. In this paper, by strengthening the continuity condition on the mapping $T$, we give a partial answer to Schauder's conjecture. To be more precise, we introduce the following concept:

Definition 1.1. Let $X$ be a topological vector space. A mapping $T: X \rightarrow X$ is said to be convex continuous at $x_{0} \in X$ if for any open neighborhood $N\left(T x_{0}\right)$ of $T x_{0}$, there exists an open neighborhood $V\left(x_{0}\right)$ of $x_{0}$ such that $\operatorname{Conv}\left(T V\left(x_{0}\right)\right) \subset$ $N\left(T x_{0}\right)$, where $\operatorname{Conv}\left(T V\left(x_{0}\right)\right)$ represents the convex hull of $T V\left(x_{0}\right)$.

It is easy to see that the constant mapping is convex continuous. One can also show that a mapping with a finite dimensional range is convex continuous. If $X$ is not locally convex, then the identity mapping on $X$ is continuous but not convex

Received by the editors July 14, 1999 and, in revised form, October 27, 1999.

2000 Mathematics Subject Classification. Primary 54H25; Secondary 47H10.

Key words and phrases. Topological vector space, convex compact set, fixed point.

(C)2000 American Mathematical Society 
continuous. Even though convex continuity is stronger, we show in section 3 that some continuous mappings can be approximated by convex continuous mappings. More precisely, we show that continuous mappings in Roberts spaces can be approximated by convex continuous mappings. We prove that Schauder's fixed point theorem is also true for a convex continuous approximatable mapping. When $X$ is a locally convex space, it turns out that these two concepts are equivalent. We have the following:

Proposition 1.1. Let $X$ be a locally convex space. Then $T: X \rightarrow X$ is convex continuous if and only if $T$ is continuous.

The proof follows from the fact that every open neighborhood of 0 contains an open convex neighborhood of 0 .

\section{SCHAUDER'S FIXED POINT THEOREM}

In the following, we always assume that $X$ is a Hausdorff topological vector space with property $(W)$ :

$X$ has a local base $\left\{W_{i}\right\}_{i \in I}$ of 0 , where $I$ is an index set with a partial order " $<$ ", such that $W_{i} \subset W_{j}$ if $i<j$ and for any $i_{1}, i_{2}, \cdots, i_{k}$, there is an $l \in\{1,2, \cdots, k\}$, such that $i_{l}=\max \left\{i_{1}, i_{2}, \cdots, i_{k}\right\}$ and $W_{i_{j}} \subset W_{i_{l}}$ for $j=1,2, \cdots, k, j \neq l$.

For example, if $X$ is first countable (equivalently, metrizable), then such a base exists.

Theorem 2.1. Let $X$ be a Hausdorff topological vector space with property $(W)$, and $C \subset X$ a convex compact subset. Suppose $T: C \rightarrow C$ is a convex continuous mapping. Then $T$ has a fixed point in $C$.

Proof. Let $\mathcal{N}=\left\{W_{i}\right\}_{i \in I}$ be the local base of 0 such that property $(W)$ holds. We may assume that $\left\{W_{i}\right\}_{i \in I}$ is a symmetric base. (Otherwise, put $W_{i}^{\prime}=W_{i} \cap\left(-W_{i}\right)$.)

For any open neighborhood $V$ of 0 and $x \in C$, by the convex continuity of $T$, there exists a $W_{i_{x}} \in \mathcal{N}$, such that $W_{i_{x}} \subset V$ and $\operatorname{Conv}\left\{T\left(x+W_{i_{x}}\right)\right\} \subset T x+V$. By continuity of addition in $X$, we may choose $W_{i_{x}}^{\prime} \in \mathcal{N}$, such that $W_{i_{x}}^{\prime}+W_{i_{x}}^{\prime} \subset W_{i_{x}}$.

Now, $\left\{x+W_{i_{x}}^{\prime}: x \in C\right\}$ is an open covering of $C$, so it contains a finite open sub-covering of $C$. We denote it by $\bigcup_{j=1}^{n}\left(x_{j}+W_{i_{x_{j}}}^{\prime}\right)$.

Let $\left\{\psi_{j}(x)\right\}_{j=1}^{n}$ be a continuous partition of unity subordinated to the covering $\left\{x_{j}+W_{i_{x_{j}}}^{\prime}\right\}_{j=1}^{n}, \sum_{j=1}^{n} \psi_{j}(x)=1, \forall x \in C$.

We define a mapping $T_{V}: C \rightarrow C$ by

$$
T_{V} x=\sum_{j=1}^{n} \psi_{j}(x) T x_{j}, \forall x \in C .
$$

Then it follows from the Brouwer fixed point theorem that $T_{V}$ has a fixed point $x_{V} \in C$. We claim that there exists $y_{V} \in C$, such that

$$
x_{V}-y_{V} \in V \text { and } x_{V}-T y_{V} \in V \text {. }
$$

Let $i_{x_{j_{0}}}=\max \left\{i_{x_{1}}, i_{x_{2}}, \cdots, i_{x_{n}}\right\}$. We may assume that $\psi_{j}\left(x_{V}\right) \neq 0, j=1,2, \cdots, n$. (Otherwise, we exclude that term.)

Since $x_{V} \in x_{j}+W_{i_{x_{j}}}^{\prime}, j=1,2, \cdots, n$, it follows that

$$
\begin{aligned}
x_{j} & \in x_{V}+W_{i_{x_{j}}}^{\prime} \subset x_{j_{0}}+W_{i_{x_{0}}}^{\prime}+W_{i_{x_{j}}}^{\prime} \subset x_{j_{0}}+W_{i_{x_{0}}}^{\prime}+W_{i_{x_{0}}}^{\prime} \\
& \subset x_{j_{0}}+W_{i_{x_{j_{0}}}}, j \neq j_{0} .
\end{aligned}
$$


Hence we have

$$
T_{V} x_{V}=\sum_{j=1}^{n} \psi_{j}(x) T x_{j} \in \operatorname{Conv}\left\{T\left(x_{j_{0}}+W_{i_{x_{0}}}\right)\right\} \subset T x_{j_{0}}+V .
$$

Let $y_{V}=x_{j_{0}}$. Then $x_{V}-y_{V} \in V, x_{V}-T y_{V} \in V$, as desired.

By the compactness of $C$, we may assume that $\left\{x_{V}, V \in \mathcal{N}\right\}$ has a subnet $\left\{x_{V^{\prime}}\right\}$ such that $x_{V^{\prime}}$ converges to $x_{0} \in C$.

By (2.1), we know that $T x_{0}=x_{0}$, i.e. $x_{0}$ is a fixed point of $T$ in $C$.

This completes the proof.

Theorem 2.1 gives a positive answer to the Schauder conjecture under the convex continuity condition. The following (almost trivial) result shows that the convex continuity is also necessary in some sense.

Theorem 2.2. Let $X$ be a Hausdorff topological vector space with property $(W)$, and $C \subset X$ a convex compact subset, $T: C \rightarrow C$ a continuous mapping. Then $T$ has a fixed point in $C$ if and only if there exists a convex compact subset $C^{\prime}$ of $C$ such that $T: C^{\prime} \rightarrow C^{\prime}$ and $T$ is convex continuous on $C^{\prime}$.

Proof. The sufficiency follows from Theorem 2.1.

For the necessity, suppose that $T$ has a fixed point $x_{0} \in C$. Set $C^{\prime}=\left\{x_{0}\right\}$. Then $T: C^{\prime} \rightarrow C^{\prime}$ is convex continuous.

\section{Convex continuous approximatable mappings}

In the following, we assume that $X$ is a topological vector space unless specified otherwise. In section 2, we proved Schauder's fixed point theorem under the convex continuity condition. However, we know that the identity mapping is not convex continuous, and more generally, the continuous open mappings are not convex continuous, so a natural question is: How large is this class of mappings? It is difficult to answer this question completely, but we can give a partial answer to some extent. We shall approximate a continuous mapping by convex continuous mappings. To be precise, we introduce the following:

Definition 3.1. Let $T: D \subseteq X \rightarrow D$ be a continuous mapping, where $D$ is a closed convex subset. If for any open neighborhood $V$ of 0 there exist a closed convex subset $D^{\prime} \subset D$ and a convex continuous mapping $T_{V}: D^{\prime} \rightarrow D^{\prime}$ such that $T x-T_{V} x \in V$ for all $x \in D^{\prime}$, then we say that $T$ is a convex continuous approximatable mapping.

Proposition 3.1. The identity mapping $I: D \rightarrow D$ of a closed convex subset $D$ in $X$ is convex continuous approximatable.

Proof. For any open neighborhood $V$ of 0 , take a finite dimensional subspace $F$ of $X$ such that $F \cap D=D^{\prime} \neq \varnothing$. Let $I^{\prime}=\left.I\right|_{D^{\prime}}$. Then $I^{\prime}: D^{\prime} \rightarrow D^{\prime}$ is convex continuous and satisfies $I x-I^{\prime} x=0 \in V$ for all $x \in D^{\prime}$. By Definition 3.1, $I$ is convex continuous approximatable.

The following result shows that Schauder's fixed point theorem still holds for convex continuous approximatable mappings.

Theorem 3.1. Let $C \subset X$ be a compact convex subset, and assume that $X$ has property $(W)$. (See section 2.) Suppose $T: C \rightarrow C$ is a convex continuous approximatable mapping. Then $T$ has a fixed point in $C$. 
Proof. For each open neighborhood $V$ of $X$, there exist a closed convex subset $C^{\prime}$ of $C$ and a convex continuous mapping $T^{\prime}: C^{\prime} \rightarrow C^{\prime}$ such that $T x-T^{\prime} x \in V$ for all $x \in C^{\prime}$.

By Theorem 2.1, $T^{\prime}$ has a fixed point $x_{V} \in C^{\prime}$. Hence we have

$$
T x_{V}-x_{V} \in V \text {. }
$$

By the compactness of $C$, we know that $\left\{x_{V}: V \in \mathcal{N}\right\}$ has a subnet $\left\{x_{V^{\prime}}\right\}$ such that $x_{V}$, converges to $x_{0}$, where $\mathcal{N}$ is a local base of 0 .

In view of (3.1), we know that $T x_{0}=x_{0}$. Therefore $T$ has a fixed point in $C$.

This completes the proof.

In the following, we show that a continuous mapping in a so-called Roberts space is convex continuous approximatable. (See [13, [18, 21], [22].) First, we recall the definition of a Roberts space as follows:

Let $X$ be a metrizable topological vector space. Then there exists a pseudonorm $\|\cdot\|$ on $X$ which is monotone, i.e. $\|s x\| \leq\|x\|$ if $s \leq 1$ (see [14, [23]), and induces an invariant metric on $X$. Let $A \subset X$ be a subset. We denote by: $A^{+}=\operatorname{conv}(A \cup\{0\}), A^{*}=\operatorname{conv}\left(A^{+} \cup\left(-A^{+}\right)\right)$.

Following 21, a non-zero point $x_{0}$ of $X$ is called a needle point if for every $\epsilon>0$ there exists a finite set $A\left(x_{0}, \epsilon\right)=\left\{x_{1}, x_{2}, \cdots, x_{n}\right\}$ satisfying the following conditions:

1. $\left\|x_{i}\right\|<\epsilon, i=1,2, \cdots, n$;

2. for each $x \in A^{+}\left(x_{0}, \epsilon\right)$, there exists an $\alpha \in[0,1]$ such that $\left\|x-\alpha x_{0}\right\|<\epsilon$;

3. $x_{0}=\frac{1}{n} \sum_{i=1}^{n} x_{i}$.

$X$ is called a needle point space if $X$ is complete metrizable and every non-zero point in $X$ is a needle point. See [13], 21] for examples.

Now assume that $X$ is a needle point space. Following Roberts' construction, take $x_{0} \neq 0$, and put $A_{0}=\left\{x_{0}\right\}$. Define a sequence $\left\{A_{n}\right\}$ of finite subsets of $X$ by induction as follows:

(4) $\|x\|<\epsilon_{n}$ for $x \in A_{n}$, where $\epsilon_{n}=\left(m_{n-1}\right)^{-1} 2^{-n}, m_{n}=\operatorname{card} A_{n}, n \geq 1$;

(5) if $a_{n}=\left\{x_{1}^{n}, x_{2}^{n}, \cdots, x_{m_{n}}^{n}\right\}$, then

$A_{n+1}=\bigcup_{i=1}^{m_{n}} A\left(x_{i}^{n}, \epsilon_{n+1}\right)$, where $A\left(x_{i}^{n}, \epsilon_{n+1}\right)$ satisfies (1)-(3) for $i=1,2, \cdots, m_{n}$.

Finally, we put $C=\overline{\bigcup_{i=1}^{\infty} A_{n}^{*}}$. Then $C$ is a compact convex set with no extreme point. See [21]. Following [18], we call $C$ a Roberts space.

Theorem 3.2. Let $T: C \rightarrow C$ be a continuous mapping, where $C$ is a Roberts space. Then $T$ is a convex continuous approximatable mapping.

Proof. Since $X$ is metrizable, we only need to show that for each $\epsilon>0$, there exists a closed convex subset $C_{\epsilon} \subset C$, and a convex continuous mapping $T^{\prime}: C_{\epsilon} \rightarrow C_{\epsilon}$ such that $\left\|T x-T^{\prime} x\right\|<\epsilon$.

We use an auxiliary mapping defined in [18]. Take $n$ sufficiently large such that $2^{-n+8}<\epsilon$. Then there exists a continuous mapping $g: A_{n}^{*} \rightarrow A_{n}^{*}$ such that $\|T x-g(x)\|<2^{-1} \epsilon$ for all $x \in A_{n}^{*}$, where $g$ is defined in the proof of Theorem 3 in [18].

Let $C_{\epsilon}=A_{n}^{*}, T^{\prime}=g$. By the construction of a Roberts space, $A_{n}^{*}$ is closed convex and contained in a finite dimensional space, so $T^{\prime}$ is convex continuous.

The proof is complete. 
In the following, we assume that $X$ is metrizable, $\Omega \subset X$ is an open nonempty subset, $T: \bar{\Omega} \rightarrow X$ is a convex continuous mapping and $T \bar{\Omega}$ is relatively compact in $X$. We will try to approximate $T$ by continuous mappings with finite dimensional range. Such an approach was used in [14], and also in [12] for the construction of the Leray-Schauder degree. See also [2].

Theorem 3.3. Suppose $T \bar{\Omega} \cap \bar{\Omega} \neq \varnothing$. Then for each $V \in \mathcal{N}$, where $\mathcal{N}=\left\{W_{i}\right\}_{i \in I}$, $I$-countable, is a local base of 0 with property $(W)$, there exist an open subset $\Omega_{V} \subseteq$ $\Omega, \overline{T \bar{\Omega}} \cap \bar{\Omega} \subseteq \Omega_{V}$, a finite dimensional subspace $F_{V}$ of $X$, and a continuous mapping $T_{V}: \Omega_{V} \rightarrow F_{V}$, such that for each $x \in \Omega_{V}$, there exists a $y^{x} \in \Omega_{V}$, such that

$$
x-y^{x} \in V, T_{V} x-T y^{x} \in V .
$$

Proof. For each $V \in \mathcal{N}, x \in \bar{\Omega}$, by the convex continuity of $T$, there exists a $W_{i_{x}} \in \mathcal{N}$ such that $\operatorname{Conv}\left\{T\left(x+W_{i_{x}}\right)\right\} \subset T x+V$. Let $W_{i_{x}}^{\prime} \in \mathcal{N}$ be such that $W_{i_{x}}^{\prime}+W_{i_{x}}^{\prime} \subset W_{i_{x}}$.

Since $\left\{x+W_{i_{x}}^{\prime}\right\}_{x \in \bar{\Omega}}$ is an open covering of $\overline{T \bar{\Omega}} \cap \bar{\Omega}$, it has a finite sub-covering $\left\{x_{j}+W_{i_{x_{j}}}^{\prime}: j=1,2, \cdots, n\right\}$.

Now, put $\Omega_{V}=\bigcup_{j=1}^{n}\left(x_{j}+W_{i_{x_{j}}}^{\prime}\right)$. Let $\left\{\psi_{j}(x), j=1,2, \cdots, n\right\}$ be a continuous partition of unity subordinated to the covering $\left\{x_{j}+W_{i_{x_{j}}}^{\prime}\right\}_{j=1}^{n}$, such that $\sum_{j=1}^{n} \psi_{j}(x)=1$. (Remark that $X$ is metrizable, so that such a partition of unity exists.)

We define the mapping $T_{V}: \overline{\Omega_{V}} \rightarrow F_{V}=\operatorname{span}\left\{T x_{j}, j=1,2, \cdots, n\right\}$ as follows:

$$
T_{V} x=\sum_{j=1}^{n} \psi_{j}(x) T x_{j}, x \in \overline{\Omega_{V}} .
$$

Following the proof of (2.1), we conclude that (3.2) is true.

\section{ACKNOWLEDGMENT}

The author would like to thank the anonymous referee for his or her valuable comments and suggestions. I am also indebted to Professors Alan Dow and Simeon Reich for their criticism, to Professor Sergiu Aizicovici for his encouragement and help.

\section{REFERENCES}

1. F. E. Browder, The fixed point theory of multivalued mappings in topological vector spaces, Math. Ann., 177 (1968), 283-301. MR 37:4679

2. K. Deimling, Nonlinear Functional Analysis, Springer-Verlag, 1985. MR 86j:47001

3. J. Dugundji, A. Granas, Fixed Point Theory, I, Warzawa, 1982. MR 83j:54038

4. S. Eilenberg and D. Montgomery, Fixed point theorems for multivalued transformations, Amer. J. Math., 58 (1946), 214-222. MR 8:51a

5. K. Fan, Fixed-point and minimax theorems in locally convex topological linear spaces, Proc. Nat. Acad. Sci. U. S. A., 38 (1952), 121-126. MR 13:858d

6. I. Glicksberg, A further generalization of the Kakutani fixed-point theorem, with application to Nash equilibrium points, Proc. Amer. Math. Soc., 3 (1952), 170-174. MR 13:764g

7. A. Granas, KKM-maps and their applications to nonlinear problems, The Scottish Book, Ed., R. D. Mauldin, Birkhauser, 1981, p. 45-61.

8. O. Hadzic, On Kakutani's fixed point theorem in topological vector spaces, Bull. Acad. Polon. Sci. Sér. Sci. Math., 30 (1982), 141-144. MR 84a:47073 
9. O. Hadzic, Fixed point theorems in not necessarily locally convex topological vector spaces, Funtional Analysis, Proc. Dubrovnik 1981, Lect. Notes in Math., vol. 948, Springer-Verlag, 1982, pp. 118-130.

10. C. J. Himmelberg, Fixed points of compact multifunctions, J. Math. Anal. Appl., 38 (1972), 205-207. MR 46:2505

11. A. Idzik, Almost fixed point theorems, Proc. Amer. Math. Soc., 104 (1988), 779-784. MR 89i: 47108

12. W. Kaballo, Abbildungsgrad in Hausdorffschen topologischen vektorraumen, Manuscripta Math., 8 (1973), 209-216. MR 50:10922

13. N. J. Kalton, N. T. Peck, A re-examination of Roberts' example of compact convex sets with no extreme points, Math. Ann., 253 (1980), 89-101. MR 82h:46055

14. V. Klee, Leray-Schauder theory without local convexity, Math. Ann., 141 (1960), 286-296. MR 24:A1004

15. M. Nagumo, Degree of mapping in convex linear topological spaces, Amer. J. Math., 73 (1951), 497-511. MR 13:150b

16. Nguyen To Nhu, The finite dimensional approximation property and the AR-property in needle point spaces, J. London Math. Soc., 56 (1997), 584-594. MR 99k:46007

17. Nguyen To Nhu, The fixed point property for weakly compact convex sets: searching for a solution to Schauder's conjecture, Topology and its appl., 68 (1996), 1-12. MR 96k:46013

18. Nguyen To Nhu and Le Hoang Tri, No Roberts space is a counterexample to Schauder's conjecture, Topology 33 (1994), 371-378. MR 95h:46014

19. S. Reich, Fixed points in locally convex spaces, Math. Z., 125 (1972), 17-31. MR 46:6110

20. S. Reich, On fixed point theorems obtained from existence theorems for differential equations, J. Math. Anal. Appl., 54 (1974), 26-36. MR 53:6373

21. J. W. Roberts, A compact convex set with no extreme points, Studia math., 60 (1977), 255266. MR 57:10595

22. J. W. Roberts, Pathological Compact Convex Sets in the Spaces $L_{p}, 0 \leq p<1$, The Altgeld Book, University of Illinois, 1976.

23. S. Rolewicz, Metric Linear Spaces, Warszawa, 1972. MR 55:10993

24. B. Rzepecki, Remarks on Schauder's fixed point principle and its applications, Bull. Acad. Polon. Sci. Sér. Sci. Math., 27 (1979), 473-480. MR 81b:47073

25. J. Schauder, Der Fixpunktsatz in Funktionalraumen, Studia Math., 2 (1930), 171-180.

26. V. M. Sehgal and E. Morrison, A fixed point theorem for mutifunctions, Proc. Amer. Math. Soc., 38 (1973), 643-646. MR 47:906

27. A. Tychonoff, Ein fixpunktsatz, Math. Ann., 111 (1935), 767-776.

28. S. S. Zhang and Y. Q. Chen, Topological degree theory and fixed point theorems in probabilistic metric spaces, Applied Math. Mechanics, 10 (1989), 495-505. CMP 21:16

29. K. Zima, On Schauder's fixed point theorem with respect to para-normed space, Comment. Math. Prace Mat., 19 (1977), 421-423.

Department of Mathematics, Ohio University, Athens, Ohio 45701-2979

E-mail address: yuqchen@bing.math.ohiou.edu

Sichuan University, Chengdu, Sichuan 610064, People's Republic of China

E-mail address: nic2601@scu.edu.cn 